Volume 2, Issue 1, May - 2021: pp: 39-46_www.ejor.sohag-univ.edu.eg

Doi: 10.21608/ejor.2021.175573

Original article

\title{
FUNCTIONAL OUTCOME OF ARTHROSCOPIC RELEASE OF EXTENSOR CARPI RADIALIS BREVIS MUSCLE IN TREATMENT OF CHRONIC LATERAL EPICONDYLITIS (TENNIS ELBOW)
}

\author{
Mohamed Mohamed, Ahmed Zaghlool ${ }^{\left({ }^{*}\right)}$, Ahmad Addosooki, Abdelrahman Khalifa \\ Orthopaedic dept, Faculty of Medicine, Sohag University, Sohag, Egypt \\ *E-mail: drabotarek@gmail.com
}

Received 3/1/2021

Accepted 10/5/2021

\begin{abstract}
The purpose of this study was to evaluate results of the release of extensor carpi radialis brevis $(E C R B)$ in tennis elbow patients. A prospective study including twenty patients with resistant chronic lateral epicondylitis was carried out in the period between June 2018 and June 2019 at Sohag University Hospital. These 20 patients underwent arthroscopic ECRB release. Evaluation of the pain and function included visual analog scale (VAS), Quickdash score, and Nirschl staging score preoperatively, postoperatively, two weeks, four weeks, ten weeks, and six months postoperatively. The patients showed highly statistically and clinically significant differences in the form of clinical improvement indicated by the level of Quickdash, VAS, and Nirschl staging scores at each time interval of the patients follow up. There was minimal postoperative pain, early rehabilitation, and early return to activity could be achieved. Arthroscopic release of the ECRB is an effective method of treatment in patients with resistant tennis elbow.
\end{abstract}

Keyword: Tennis elbow, Arthroscopy, Release

\section{Introduction}

Lateral epicondylitis, initially named "tennis elbow," influences somewhere in the range of $1 \%$ and $3 \%$ of the populace. Around 100 years ago the condition portraying latral elbow torment in yard tennis players was named "tennis elbow" [1]. In the beginning, the condition was believed to be an inflammatory condition however with additional investigations of histological changes that happen in the ERCB tendon; scientists found that it is degenerative changes followed by repa- rative cycles as opposed to inflamematory conditions [2]. The term tendinosis instead of tendinitis was discovered better to describe vascular and fibroblastic changes that happen in the tendon, pathologically known as angiofibroblastic degeneration [3]. Likewise, the high unconventional and concentric pressure that influences the ERCB ligament prompts micro tears as laser diffraction examination of the ECRB tendon during elbow flexion showed that the sarcomere length 
changed differentially, consistent with eccentric contractions and produced powerful stress on the ECRB origin [4]. Traumatic irritation of the encompassing periosteum was likewise thought to partake in the pathology of the condition in addition to mechanical changes that outcome from degeneration of close to the ligamentum annular radii and the lateral condyle [5]. The most acknowledged clarification of tennis elbow was that given by Nirschl and Petrone [6] which recommended that the condition was brought about by a microscopic rupture joined by the development of reparative tissue in the extensor carpi radialis brevis origin on the lateral epicondyle. The primary line of treatment of persistent lateral elbow tendinosis should be conservative. Activity modification, rehabilitation, and local or systemic anti-inflammatory drugs are the main treatment alternatives. Additionally, physical therapy and physiotherapy can be of worth particularly a daily static stretching program [7,8]. The majority of the patients normally react to conservative therapy as it is a self-limiting condition yet generally around 5 to $10 \%$ of patients fail to respond to conservative treatment and develop chronic lateral epicondylitis [8-10]. In those individuals who fail to respond to the variety of treatment alternatives, surgery is the subsequent stage in therapy to eliminate the pathological tissue with various methodologies either open or mini open or arthroscopic surgery [11-15] with various strategies for every one of them. In the present study, we attempted to assess clinical outcome of arthroscopic release of ERCB.

\section{Patients and Methods}

During the period between June 2018 and June 2019 in the orthopedic and traumatology department in Sohag University hospital, twenty patients diagnosed with recalcitrant lateral epicondylitis underwent arthroscopic treatment. Twenty patients (9 men and 11 women) underwent (ECRB) release. The mean age of patients was 39.3 (range 24-58) years. The evaluation and follow-up were done preoperatively, postoperatively, two weeks, four weeks, ten weeks, and six months postoperatively. Before undergoing arthroscopic surgery, all patients were initially treated with a minimum of 6 months of non operative treatment. Conservative modalities consisted of relative rest, activity modification, non steroidal anti-inflammatory medication, counterforce bracing, corticosteroid injections, and physical therapy. Inclusion criteria were that the patients needed to present with lateral epicondylitis for which conservative treatment had failed over more than six months and age from 15 to 60 years old. Exclusion criteria were previous surgery on the ipsilateral elbow, previous fractures on the ipsilateral elbow, presence of concomitant disorders, such as lateral compartment arthrosis, posterior interosseous syndrome, osteochondritis dissecans of the capitellum, instability and rheumatological diseases, and patients with bleeding disorders such as Hemophilia A and B, Factors II, V, VII, X, XII deficiencies. The subjective evaluation included the assessment of pain level using a visual analog scale (VAS), Nirschl staging score, and evaluation of function using Quickdash score preoperatively, postoperatively, two weeks, four weeks, ten weeks, and six months postoperatively. 


\subsection{Surgical technique}

All the procedures were performed with the patients positioned in the supine decubitus position under general anesthesia. Elbow support attached to the operating table was positioned under the arm, allowing for the elbow to be moved from $90^{\circ}$ of flexion to full extension, fig. (1).

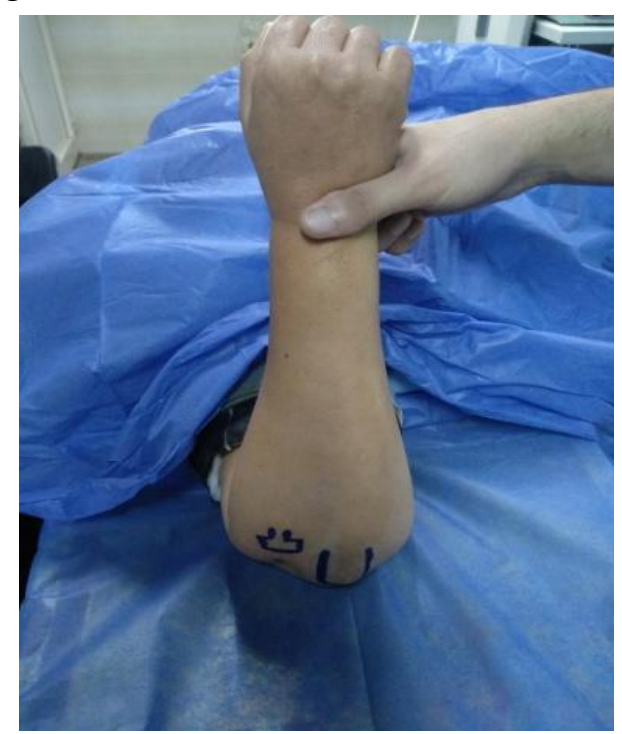

Figure (1) shows supine decubitus

The bony landmarks; medial epicondyle, lateral epicondyle, olecranon tip, radial head, and ulnar nerve are palpated and outlined; then portal localization is done. They were marked with a marker, fig. (2).

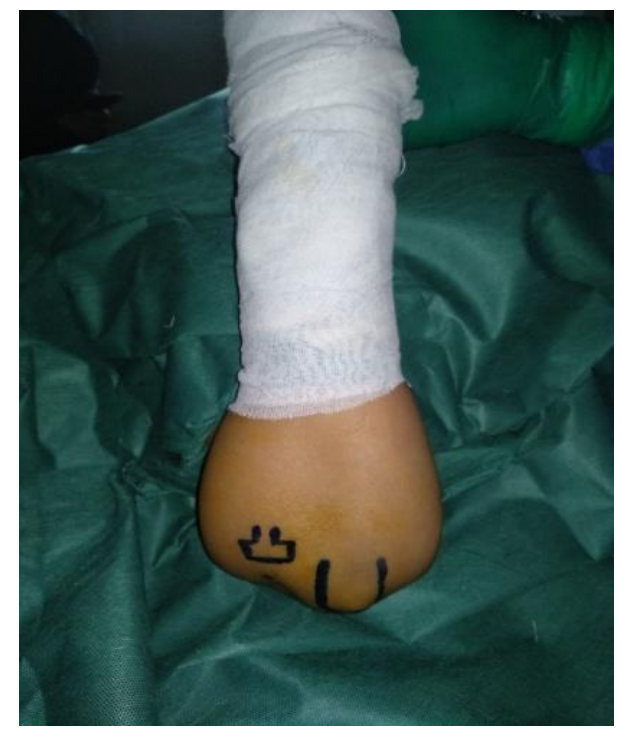

Figure (1) shows anatomical landmarks
After the landmarks have been identified and marked on the skin, the limb was exsanguinated, and the tourniquet was inflated to $250 \mathrm{mmHg}$. An 18 gauge syringe needle was introduced into the lateral soft spot which can be found in the center of a triangle formed by the palpable radial head, lateral epicondyle, and olecranon. The joint was injected with approximately 20 to $30 \mathrm{~mL}$ of saline, fig. (3).

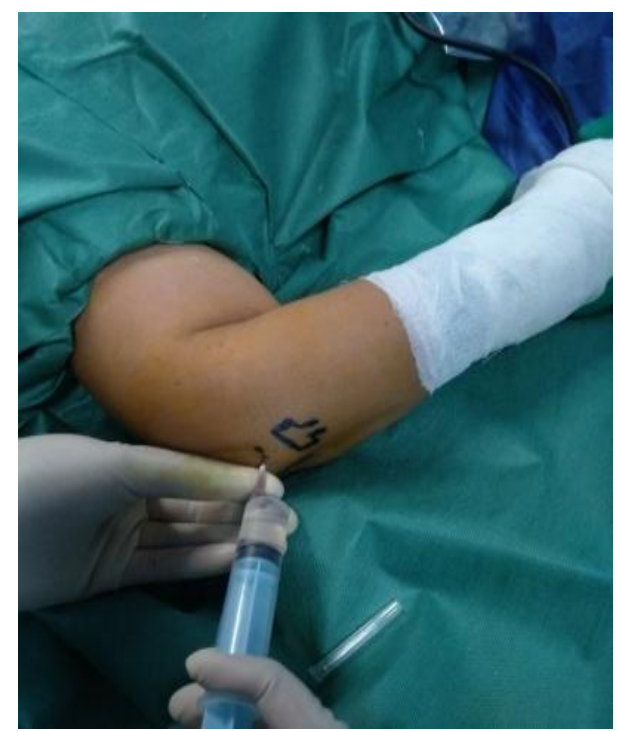

Figure (3) shows insufflation of the joint

Joint distention pushes the neurovascular structures more anterior, thereby protecting them from iatrogenic injury. Free backflow of fluid confirms correct intraarticular placement of the needle. The proximal anterolateral portal $(2 \mathrm{~cm}$ proximal and $1 \mathrm{~cm}$ anterior to the lateral epicondyle) was identified (working portal). The skin was incised with the tip of a No.11 blade by pulling the skin against the cutting edge. A mosquito hemostat was used to dissect bluntly down to the fascia to minimize the chance of injury to cutaneous or radial nerves; aiming the mosquito towards the center of the joint. Then the following stru-ctures were examined: medial joint 
capsule, trochlea, coronoid fossa, and coronoid process. A blunt trocar was inserted through the portal and aimed toward the center of the joint to maintain contact with the anterior surface of the humerus to identify the proximal anteromedial portal. Then, the proximal anteromedial portal (viewing portal) was created approximately $2 \mathrm{~cm}$ proximal to the medial epicondyle and just anterior to the medial intermuscular septum, fig. (4).

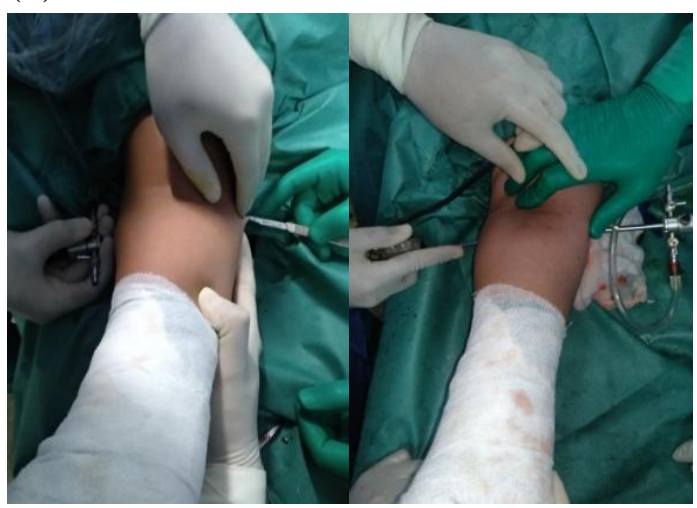

Figure (4) shows transfer scope from lateral to medial portal

To protect against injury to the sensory nerves, the nick and spread technique were used, in which only the skin was incised, and a mosquito hemostat was used to spread the subcutaneous tissues. The $4 \mathrm{~mm}, 30^{\circ}$ arthroscope was inserted into the joint. The lateral capsule and radioca-pitellar articulation were easily inspected. From this portal, the following structures were examined: capitellum, radial head, anterior and lateral joint capsule, coronoid fossa, and coronoid process. The radiocap-itellar articulation was examined with the arm in pronation and supination. The joint capsule was examined for thickening, scarring, and inflammation. After a diagnostic arthroscopy which allowed visualization of the entire anterior aspect of the elbow and a perfect evaluation of the lateral structures, the area of damage to the ECRB was identified. Then, the shaver was then placed into the proximal anterolateral portal, and the portion of the lateral capsule was resected and revealed the underlying common extensor origin. The shaver was exchanged for a monopolar radiofrequency device. The ECRB origin was then completely released from its insertion, and the tendinosis tissue ablated until only healthy tendon remained or the ECRL tendon appeared, fig. (5).

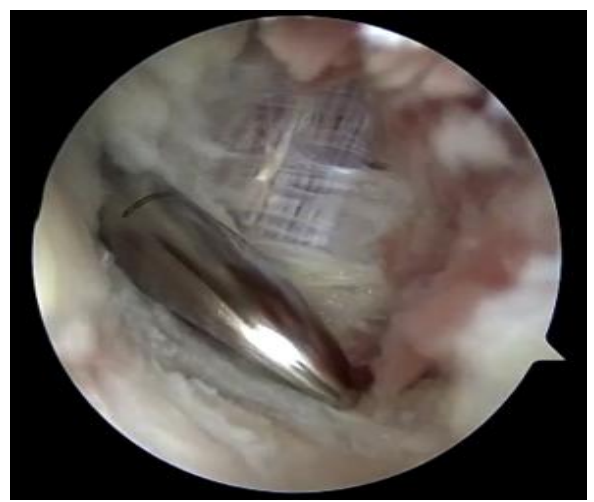

Figure (5) shows ERCB release

To protect the lateral ligamentous structures, care was taken not to extend the release posterior to a line bisecting the radial head and not to extend beyond the radial head distally to avoid PIN injury. The portal sites were closed with simple sutures, and a sterile dressing was applied.

\subsection{Statistical analysis}

The collected data was revised, coded, tabulated, and analyzed using the Statistical Package for Social Science (SPSS 25). Data were presented and suitable analysis was done according to the type of data obtained for each parameter.

\subsubsection{Descriptive statistics}

Mean, standard deviation $( \pm \mathrm{SD})$, and range for parametric numerical data, while Median for non-parametric numerical data. Frequency and percentage for nonnumerical data. 


\subsubsection{Analytical statistics}

ANOVA test was used to assess the statistical significance of the difference between means.

\section{Results}

The patients underwent arthroscopic release of ERCB showed highly statistically significant differences between Preoperative and postoperative data in the following parameters: level of Quickdash, VAS and Nirschl staging scores at each time interval of the patients follow up, the results are listed in tab $(1,2,3)$.

Table (1) Nirschl staging score

\begin{tabular}{|c|c|c|c|c|}
\hline & Mean & SD & Range & $\begin{array}{c}\text { One Way ANOVA } \\
\text { test of sig. }\end{array}$ \\
\hline Preoperative & 4.60 & 1.90 & $(2-7)$ & \multirow{6}{*}{$<0.001^{*}$} \\
\hline Post- operative & 4.30 & 1.42 & $(3-7)$ & \\
\hline 2 weeks & 3.10 & 1.37 & $(1-5)$ & \\
\hline 4 weeks & 2.60 & 1.17 & $(1-5)$ & \\
\hline 10 weeks & 2.30 & 0.82 & $(1-4)$ & \\
\hline 6 months & 1.70 & 1.06 & $(1-4)$ & \\
\hline
\end{tabular}

Table (2) visual analogue score (VAS)

\begin{tabular}{|c|c|c|c|c|}
\hline & Mean & SD & Range & $\begin{array}{l}\text { One Way ANOVA } \\
\text { test of sig. }\end{array}$ \\
\hline Preoperative & 5.40 & 1.90 & $(3-8)$ & \multirow{6}{*}{$<0.001^{*}$} \\
\hline Post-operative & 6.00 & 2.05 & $(3-9)$ & \\
\hline 2 weeks & 4.20 & 1.81 & $(1-6)$ & \\
\hline 4 weeks & 3.40 & 1.78 & $(0-6)$ & \\
\hline 10 weeks & 2.80 & 1.69 & $(0-6)$ & \\
\hline 6 months & 1.90 & 1.73 & $(0-5)$ & \\
\hline
\end{tabular}

Table (3) quick DASH score

\begin{tabular}{|c|c|c|c|c|}
\hline & Mean & SD & Range & $\begin{array}{l}\text { One Way ANOVA } \\
\text { test of sig. }\end{array}$ \\
\hline Preoperative & 23.80 & 8.72 & $(12-38)$ & \multirow{6}{*}{$<0.001^{*}$} \\
\hline Post-operative & 31.80 & 6.96 & $(25-45)$ & \\
\hline 2 weeks & 17.90 & 4.58 & $(10-24)$ & \\
\hline 4 weeks & 13.90 & 4.98 & $(2-20)$ & \\
\hline 10 weeks & 10.60 & 4.58 & $(1-18)$ & \\
\hline 6 months & 6.10 & 3.70 & $(0-12)$ & \\
\hline
\end{tabular}

\section{Discussion}

In the literature, many different surgical techniques were described for management of tennis elbow. These techniques include simple release, reconstruction of the common extensor tendons, a separation of the deep fascia that covers the common extensor tendon and arthroscopic release and repair of lesions [16]. Baker and Cummings [17] described their experie-nce with arthroscopic treatment of lateral epicondylitis in 1998. Their technique involved excision of the lateral capsule, debridement of any pathologic tendon tissue on the undersurface of the ECRB, and decortication of the lateral epicondyle. They reported that overall, 93.3\% of their patients improved, and $71 \%$ of the patients had good or excellent results. Also they noted that patients returned to full functional activity at an average of 35 days compared with an average of 2.6 months in the study of Nirschl and Pettrone [18]. In 2013, Solheim et al [19], in their study comparing between arthroscopic and open tennis elbow release concluded that; both a traditional open approach and the newer arthroscopic method provide an effective treatment of recalcitrant tennis elbow without major complications. The arthroscopic method offers a small, but not insignificant, improvement in the outcome as evaluated by the Quick-DASH score.

\subsection{The Quick DASH score}

Quick-DASH score quantifies pain and disability related to the upper extremity and ranges between 0 and 100 points. The lower DASH scores represent less pain and disability. A study on a non clinical population [20] indicated that the DASH score in healthy employed adults is $13 \pm 1.5$. Thus, in this study, a DASH score below 14 points was considered a good result. In our study DASH score improved from a mean of 23.8 points pre operative to 6.1 points at six months postoperative, indeed this result was somewhat good result in comparison to others studies. Wada et al. [21], in their study; results showed that the mean postoperative DASH score 10.6 points (range: 0-50). Othman [22], in his study 
on 33 patients comparing the arthroscopic versus percutaneous release in treatment of chronic tennis elbow, noticed that the mean DASH score of the 14 patients who underwent arthroscopic release, improved from 72 points to 48 points postoperatively and he concluded that the arthroscopic treatment of lateral epicondylitis gives more favorable results in comparison to percutaneous one. Rhyou and Kim [23], in their retrospective study on 39 patients who underwent arthroscopic treatment for refractory lateral epicondylitis from November 2003 to October 2009. They grouped 20 patients treated with arthroscopic release of the ECRB origin. They noted that the mean DASH score in improved from 54.6 points to 6.1 points.

\subsection{The Visual analog score (VAS)}

VAS in the current study: the mean improved from mean 5.4 points preoperative (range: 3 and 8 ) to mean 1.9 points (range: 0 and 5) at six months postoperative. We found comparable results in the other studies. Owens et al [24], in their retrospective review of 16 patients with lateral epicondylitis treated with an arthroscopic release of the ECRB between Jan. 1995 and Nov. 1998 with a minimum of 1-year clinical follow-up, they noted that the mean postoperative VAS was 0.58 point (range: 0-3). Szabo et al [25], in their study that evaluated three surgical methods for treatment of lateral epicondylitis, they found that the mean VAS in arthroscopic group improved from 5.2 points to 1 point postoperatively. Baker and Baker [26], in their recent study conducted on 30 patients were evaluated at a mean of 130 months after surgery (range, 106-173 months). They noted that the mean VAS was 0.

\subsection{The Nirschl staging score}

The Nirschl staging score in our study improved from mean 4.6 points preoperative with range (2-7 points) to mean 1.7 points with range (1-4 points) at six months postoperative. Our present study had: some points of strengths that operation was done by the same surgical team, the results were analyzed by an independent investigator to avoid investigator bias and the clinical and functional outcome was determined based on standard scores while the points of weakness were the relatively small sample size.

\section{Conclusion}

Through the data mentioned in the previous study, it could be concluded that the arthroscopic surgery approach is a successful treatment of chronic lateral epicondylitis with good clinical outcome results and early return to work

\section{References}

[1] Major, H. (1883). Lawn-tennis elbow. Br Med J. 2: 557

[2] Beckert, A., Biskop, M., Clemen, G. (1981). Diagnostik und therapie der epicondylitis humeri-eine gemeinschaftsstudie. Orthop Traumatol. 28: 278-287

[3] Almekinders, L., Baynes, A., Bracey, L. (1995). An in vitro investigation into the effects of repetitive motion and nonsteroidal anti-inflammatory medication on human tendon fibroblasts. Am J. Sports Med. 23:119-123

[4] Lieber, R., Loren, G., Friden, J. (1994). In vivo measurement of human wrist extensor muscle sarcomere length changes. J. Neur-ophysiol. 71: 874881

[5] Bosworth. D. (1995). The role of the orbicular ligament in tennis elbow. J. Bone Joint Surg Am. 37:527-533 
[6] Nirschl, R., Pettrone, F. (1979). Tennis elbow: The surgical treatment of lateral epicondylitis. J. Bone Joint Surg Am. 61: 832-839

[7] Coonrad, R., Hooper, W.(1973). Tennis elbow: its course, natural history, conservative and surgical management. J. Bone Joint Surg Am. 55: 11771182

[8] Nirschl, R. (1988). Prevention and treatment of elbow and shoulder injuries in the tennis player. Clin Sports Med. 7: 289-308

[9] Cyriax, J. (1936). The pathology and treatment of tennis elbow. J. Bone Joint Surg. 18: 921-940

[10] Kuklo, T., Taylor, K., Murphy, K., et al. (1999). Arthroscopic release for lateral epicondylitis: a cadaveric model. Arthroscopy. 15: 259-264

[11] Terra, B., Rodrigues, L., Filho, A., et al. (2015). Arthroscopic treatment for chronic lateral epicondylitis. Rev Bras Ortop. 50 (4): 395-402.

[12] Ortega J., Apóstol-González, S., Pizzolla, P. (2019). Arthroscopic treatment of chronic lateral epicondylitis. Short-termresults of 3 cases, Acta Ortop Mex. 33 (1): 24-27.

[13] Moradi A., Pasdar, P., Mehrad-Majd., H., et al. (2019). Clinical Outcomes of open versus arthroscopic surgery for lateral epicondylitis, evidence from a systematic review. Arch Bone Jt Surg. 7 (2): 91-104.

[14] Field, L.. (2018). Editorial commentary: Dealer's choice for arthroscopic versus open lateral epicondylitis release? It's not that simple. Arthroscopy. 34 (12): 3185-3186.

[15] Clark, T., McRae, S., Leiter, J., et al. (2018). Arthroscopic versus open lateral release for the treatment of lateral epicondylitis: A prospective randomized controlled trial. Arthroscopy. 34 (12): 3177-3184.

[16] Jerosch, J., Schunck, J. (2006). Arthroscopic treatment of lateral epicondylitis: Indication, technique and early results. Knee Surg Sports Traumatol Arthrosc. 14(4): 379-382.

[17] Baker Jr, C., Cummings, P. (1998). Arthroscopic management of miscellaneous elbow disorders. Oper Tech Sports Med. 6: 16-21

[18] Smidt, N., Lewis, M., Van Der Windt, D., et al. (2006). Lateral epicondylitis in general practice: Course and prognostic indicators of outcome. J. Rheumatol. 33 (10): 2053-2059

[19] Solheim, E., Hegna, J., Oyen, J. (2013). Arthroscopic versus open tennis elbow release: 3 to 6 year results of a case-control series of 305 elbows. Arthroscopy 29 (5): 854-859.

[20] Jester, A., Harth, A., Germann, G. (2005). Measuring levels of upper extremity disability in employed adults using the DASH Questionnaire. J. Hand Surg Am. 30 (5): 1074. e1-1074.e10.

[21] Wada, T., Moriya, T., Iba, K., et al. (2009). Functional outcomes after arthroscopic treatment of lateral epicondylitis. J. Orthop Sci. 14 (2): 167-174.

[22] Othman, A. (2011). Arthroscopic versu percutaneous release of common extensor origin for treatment of chronic tennis elbow. Arch Orthop Trauma Surg. 131(3): 383-388.

[23] Rhyou, I., Kim, K. (2013). Is posterior synovial plica excision necessary for refractory lateral epicondylitis of the elbow?. Clin Orthop Relat Res. 471(1): 284-290. 
[24] Owens, B., Murphy, K., Kuklo, T. (2001). Arthroscopic release for lateral epicondylitis. Arthroscopy. 17 (6): 582-587.

[25] Szabo, S., Savoie 3rd, F., Field L., et al. (2006) Tendinosis of the extensor carpi radialis brevis: An eval- uation of three methods of operative treatment. J. Shoulder Elbow Surg. 15 (6): 721-727.

[26] Baker Jr, C., Baker 3rd, C. (2008). Long-term follow-up of arthroscopic treatment of lateral epicondylitis. Am J. Sports Med. 36: 254-260. 